\title{
Control of Multifunctional Prosthetic Hands by Processing the Electromyographic Signal
}

\author{
M. Zecca, S. Micera, M. C. Carrozza, \& P. Dario \\ ARTS Lab, Scuola Superiore Sant'Anna, Pontedera, Italy \\ Address all correspondence to Silvestro Micera, PhD, ARTS Lab, Scuola Superiore Sant'Anna, Polo Sant'Anna \\ Valdera, Viale Rinaldo Piaggio, 34, 56025 Pontedera (PI), Italy; micera@sssup.it
}

\begin{abstract}
The human hand is a complex system, with a large number of degrees of freedom (DoFs), sensors embedded in its structure, actuators and tendons, and a complex hierarchical control. Despite this complexity, the efforts required to the user to carry out the different movements is quite small (albeit after an appropriate and lengthy training). On the contrary, prosthetic hands are just a pale replication of the natural hand, with significantly reduced grasping capabilities and no sensory information delivered back to the user. Several attempts have been carried out to develop multifunctional prosthetic devices controlled by electromyographic (EMG) signals (myoelectric hands), harness (kinematic hands), dimensional changes in residual muscles, and so forth, but none of these methods permits the "natural" control of more than two DoFs. This article presents a review of the traditional methods used to control artificial hands by means of EMG signal, in both the clinical and research contexts, and introduces what could be the future developments in the control strategy of these devices.
\end{abstract}

KEYWORDS: electromyographic (EMG) signal, EMG-based control, hand prosthesis

\section{INTRODUCTION}

A continuous challenge for scientists and engineers is to replicate the sensory-motor function of the human hand, a complex and adaptable system capable of both delicate and precise manipulation and power grasping of heavy objects. ${ }^{1,2}$ This result is achieved by a combination of a large number of degrees of freedom (DoFs), proprioceptive and exteroceptive sensors, and a complex hierarchical architecture control. ${ }^{3}$ However, despite this complexity, the efforts required to the user during the daily activities are very small, even if this ability is achieved only after several years of unconscious and conscious training.

On the contrary, current commercial prosthetic hands, aimed at replicating the natural system, are unable to provide enough grasping functionality or to deliver sensory-motor information to the user. ${ }^{4-6}$ Commercially available prosthetic devices, such as Otto Bock SensorHand, ${ }^{7}$ (Otto Bock HealthCare GmbH, Duderstadt, DE)as well as multifunctional hand designs are far from providing the manipulation capabilities of the human hand. ${ }^{8}$ Moreover, for many reasons, they require a great deal of training and concentration in order to be effectively used. For example, in prosthetic hands active bending is restricted to two or three 


\section{ZECCA ET AL.}

joints, actuated by a single motor drive acting simultaneously on the metacarpo-phalangeal (MP) joints of the thumb, index, and middle finger, while other joints are fixed.

Prosthetic hands, nowadays, have optimal reliability and robustness, but at present many limitations which can be summarized, as follows':

- The low grasping capabilities, because current prosthetic hands have no more than two active DoFs (and act like a simple gripper).

- The noncosmetic and unnatural appearance of the grasping movement resulting from the low number of DoFs. On the other hand, cosmetic devices have no active functionality and can be used only as a passive support.

- The lack of sensory information given to the user. There is no feedback except visual from the outside, so the user has to judge by sight when to stop moving the hand. Otto Bock recently introduced to the market the SUVA Hand, which uses a force sensor in order to optimize the grip strength, but there is still no sensory feedback besides direct visualization and such subtle clues as the sound of the speed changes of the motor and transmission.

- The lack of a "natural," intuitive, nonfatiguing command interface, to enable practical long-term use of a multifunctional prosthetic hand.

According to the "mechatronic" design paradigm, the first two limitations could be overcome by a complete redesign of the hand prosthesis, for example using microactuators ${ }^{10,11}$ or underactuated mechanisms. ${ }^{12,13}$

Figure 1 illustrates the schematic diagram of a multifunctional hand prosthesis. Even if we could increase the number of DoFs of the prosthesis, the main limitation would remain the control of the artificial device. In fact, many DoFs cannot be controlled directly by the subject except by using complicated coding of movements, which, in turn, requires a high level of training. The user interface, on the other hand, should be as intuitive and nonfatiguing as possible to enable practical long-term use of the device, because users cannot be productive if they must spend a large portion of their energy and concentration controlling the artificial hand. ${ }^{14}$

Several possibilities were exploited over the past years. For examples, hand prosthesis could be controlled by harness (body-powered), by Tendon Activated Pneumatic (TAP) foam

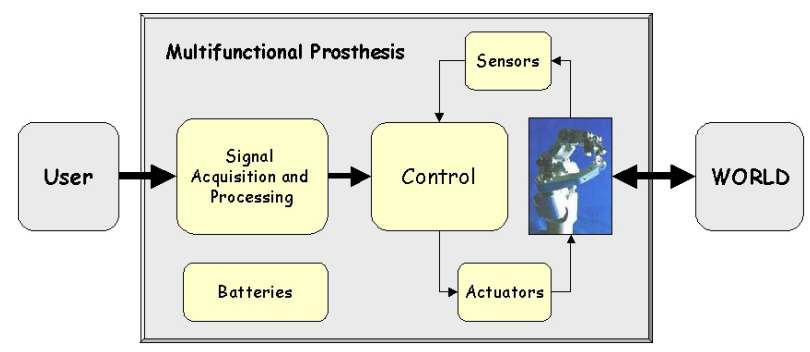

FIGURE 1. A multifunctional hand prosthesis is a mechatronic device composed by several modules: signal acquisition and processing; control; sensors; actuators; batteries. 
sensors, ${ }^{15,16}$ or by Hall effect sensors. ${ }^{17}$ Another approach uses direct tunnel muscle cineplasties, ${ }^{18,19}$ in an extension of the extended physical proprioception (EPP) concept. $^{20}$

Electromyographic (EMG) signals, collected at the skin surface, have been used for the control of upper limb prosthetic devices since $1948,{ }^{21}$ because they provide easy and noninvasive access to physiological processes that cause the contraction of the muscles. At present, the process of EMG signals is the most common approach used for the control of active prosthetic hands. In any case, the myoelectric signal permits the control of no more than one or two active DoFs (generally, one DoF for the gripper and one for the wrist). Limitations in the mechanics of the prosthetic device and in the processing of EMG data make it impossible to control more. ${ }^{22}$

\section{I.B. Aim of This Article}

In past decades, and especially during the last years, many efforts have been carried out in order to implement effective control algorithms based on the processing of EMG signals. Starting from the first attempts in the late 1940s, ${ }^{21}$ several EMG-based algorithms have been developed and used to enhance the functionality and usability of prosthetic hands.

The goal of this article is to review the state of the art of EMG-based control of artificial hands and attempt to define the potentialities and limits of this approach. The article is organized as follows: in Section II, the formal scheme of EMG acquisition and processing is presented, showing how EMG signal is acquired and processed in order to control traditional prostheses and paying particular attention to the analysis of the recent advanced techniques developed to control multifunctional prostheses. An overview of the clinical approach to the prosthetization of a user is also presented. The acquisition and processing of the EMG signal is discussed in Section II.B; the extraction of EMG features, both in the time domain and in the frequency domain, in Section II.C. The dimensionality reduction is presented in Section II.D; some techniques of pattern classification are illustrated in Section II.E; and a brief overview of online and offline learning techniques is given in Section II.F.

Finally, in Section III the merits and the limitations of these algorithms are analyzed, and possible solutions to overcoming these limits are discussed in Section IV.

\section{ACOUISITION AND PROCESSING OF EMG SIGNAL- FORMAL SCHEME}

The formal scheme for the acquisition and analysis of the EMG signal for the control of prosthetic devices is composed of several modules (Fig. 2):

- signal conditioning and preprocessing

- feature extraction

- dimensionality reduction

- pattern recognition

- offline and online learning 


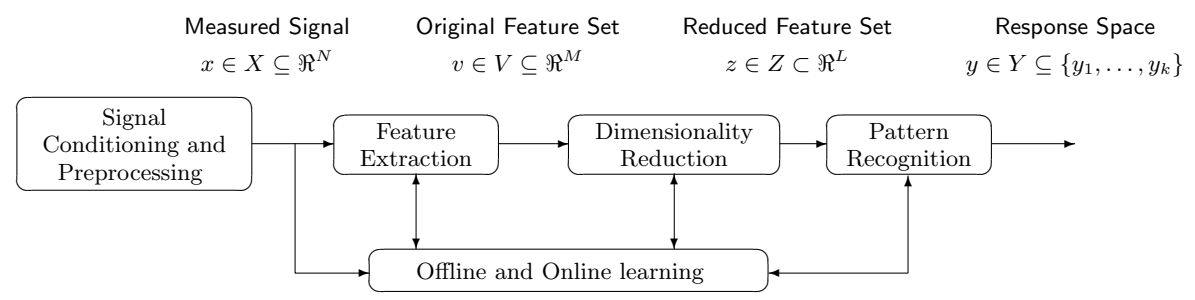

FIGURE 2. Formal scheme for acquisition and analysis of EMG for control of prosthetic devices.

The first module preprocesses the EMG signal in order to reduce noise artifacts and/or enhance spectral components that contain important information for data analysis. Moreover, it detects the onset of the movement and activates all the following modules.

During the feature extraction phase, the measured EMG signal $x \in \mathrm{X} \subseteq \mathfrak{R}^{\mathrm{N}}$ is processed in order to emphasize the relevant structures in the data, while rejecting noise and irrelevant data, producing the so-called "original feature set" $v \in \mathrm{V} \subseteq \mathfrak{R}^{\mathrm{M}}$. Sometimes a reduction of the dimensionality is needed to simplify the task of the classifier. In this case, a pattern recognition algorithm is used on the (reduced) feature set $z \in Z \subseteq \mathfrak{R}^{\mathrm{L}}$, and the measured signal is classified into the output space $y \in Y=\left\{y_{1}, \ldots, y_{\mathrm{k}}\right\}$. The learning modules are used to adapt the device to the EMG signals generated by the users because of its time-variant characteristics.

The state of the art in each of these subtopics is briefly summarized below.

\section{II.A. Preparation of Patient for Myoelectric Device}

In clinical practice, several phases are usually implemented in order to maximize the efficacy of this rehabilitation problem. ${ }^{23-25}$

1. The first step is the patient evaluation, including observation of skin condition, tissue condition, skeletal anatomy, muscle strength, range of motion, EMG testing, and contralateral side involvement. After this phase, the design of the socket-prosthesis interface and of the primary and secondary control schemes are investigated. Suspension and cosmesis can be formulated by combining data collected throughout the evaluation with knowledge of design theory.

2. The diagnostic phase begins with obtaining a plaster impression of the patient's residual limb, taking care to prepare the patient both physically and psychologically for the procedure. Some considerations about interface material, donning and doffing, and suspension have to occur prior to modification, because they will dictate modification requirements. After this phase, definition of an effective interface from both the static and dynamic points of view must be considered.

3. During the static diagnostic analysis, auxiliary suspension is included if dictated by the 
initial strategy. This is an important factor because the interface to skin contact can often change once auxiliary suspension is incorporated. Several modifications to the interface and auxiliary suspension may be required to obtain a static, total-contact, comfortable interface. Once an acceptable static interface has been achieved, dynamic diagnostic analysis follows, which insures maximum range of motion with minimal skinto-interface contact loss.

If myoelectric control is selected as the primary control scheme, site identification must consider EMG signal level, EMG separation, and skin condition. Marking an area on the skin surface that has acceptable EMG signal strength and separation and then donning the interface and transferring this site provides the best results. Once electrodes are mounted into the diagnostic interface, an EMG analyzer is attached to insure that the tissue containment strategy of the interface does not adversely effect EMG signal strength and separation, in both static and dynamic conditions. Then, when the interface is under load, reevaluation of the interface takes into account donning/doffing effort, contralateral limb involvement, comfort, range of motion, stabilization, electrode site contact, suspension, alignment, and cosmesis.

Initial prosthetic training includes basic operation instruction, care, and maintenance. Initial system optimization occurs during this phase. Evaluation of the patient's function, comfort, and cosmesis have to be included in the postdelivery evaluation plan and communicated to the rehabilitation team to insure efficient transition.

\section{II.B. EMG Acquisition and Preprocessing}

Precise detection of discrete motor events, such as the onset of voluntary muscle contractions, is a prerequisite for various psychophysiological approaches in sensorimotor system analysis. ${ }^{26,27}$ EMG signal for prosthetic applications is generally acquired by placing one or more differential electrodes on the skin of the user, depending on his/her level of amputation and on the data that should be extracted from the signal. Recently, the Surface EMG for Non-Invasive Assessment of Muscles (SENIAM) project ${ }^{28}$ defined guidelines for guaranteeing a useful exchange of knowledge and experience among scientists on the choice of electrodes, electrode placement procedures, and signal processing methods. A good acquisition of the EMG signal, in fact, is a prerequisite for good signal processing. In particular, the SENIAM consortium defined some recommendations about electrode shape and size, interelectrode distance, electrode material, and sensor construction (where sensor is defined as the ensemble of electrodes, electrode construction, and integrated preamplifier, if any).The use of one or more low-noise, high-input impedance amplifiers to acquire the EMG signal is suggested.

After the acquisition, the signal is filtered, generally using a band-pass filter with high CMRR and gain in order to reduce motion artifacts (high-pass filter) and noise (low-pass filter). Generally, about $95 \%$ of the power spectrum of the EMG is accounted for by harmonics up to $400 \mathrm{~Hz}$, and most of the remaining is electrode and equipment noise. A low-pass filter, or anti-aliasing, is usually applied to the signal. The cut-off frequency varies from 250 to $2000 \mathrm{~Hz}$, the most common choice being around $500 \mathrm{~Hz} \cdot{ }^{29} \mathrm{~A}$ high-pass filter is also required to attenuate movement artifacts and the instability of the electrode-skin interface. In the literature, the lower cut-off frequency varies from 0.1 to $100 \mathrm{~Hz}$, but generally a value between 10 and $20 \mathrm{~Hz}$ is used. In simple on-off devices a notch filter at 50 or $60 \mathrm{~Hz}$ (depending on the frequency of the electric power supply) could be added. However, it is 


\section{ZECCA ET AL.}

worth noting that this filter could also eliminate some important information present in the EMG signal and should not be used for multifunctional hands.

At this stage the signal is sampled and converted into a digital stream of data.

\section{Detection of Onset of Movement}

The first problem is the correct detection of the onset of the movement. ${ }^{26,30,31} \mathrm{In}$ fact, because of the stochastic characteristic of the surface electromyogram, onset detection is a challenging task, especially when surface EMG response is weak. Several methods and algorithms have been proposed in the literature, ${ }^{26}$ but little is known about the reliability and accuracy of these methods. The formal detection scheme is divided into two parts: the first roughly detects the presence of an event by applying a test function $g\left(x_{1}, 1 / 4 \ldots, x_{k}\right)$ to the conditioned signal; the second refines the estimation of the exact change time $t_{0}$. Staude et al. ${ }^{30}$ tested and compared the performance of different onset detection methods. The results were that some of the threshold-based signal-power estimation procedures were very sensitive to signal parameters, whereas statistically optimized algorithms were generally more robust. In particular, the generalized likelihood ratio $(\mathrm{GLR})^{30,31}$ method seems to be more robust than the other methods.

An example of a simple processing of EMG signal is given in Figure 3. The signal acquired from electrodes (Fig. 3a) is rectified (Frg. 3b) and low-pass filtered (Fig. 3c). This signal is then compared to a threshold (Fig $3 \mathrm{~d}$ ). The choice of the threshold clearly affects the performance of the detection: a lower threshold (dashed line) is more sensitive to noise, a higher one could be less precise in detecting fine movements.

\section{II.C. EMG Feature Extraction}

Many EMG-based control systems are able to control a single DoF in a prosthetic limb (hand open/close, wrist or elbow flexion/extension). These systems generally extract the EMG amplitude or rate of change by using two electrodes placed on two antagonist muscles (e.g., biceps and triceps brachii or flexor and extensor of the forearm, depending on the level of the amputation). ${ }^{22}$ This information is used to define the state of the hand and to control its speed or strength in a constant or even proportional way.

Starting from the late 1970s, the EMG signal was modeled as amplitude modulated Gaussian noise whose variance was related to the force developed by the muscle. As a consequence, most commercial myoprocessors used in prosthetic control are now based only on one dimension of the EMG signal - the variance or mean absolute value. ${ }^{32}$ The Otto Bock SUVA Hand, ${ }^{7}$ mentioned above, is designed so that the grip speed and grip force are controlled by the intensity of the muscle signal. Two independent measurements and control systems ensure that the hand switches to grip force mode when an object is gripped and the grip force is proportional to the muscle signal.

Several authors successfully contributed in refining variance estimation from the myoelectric signal, for example by applying a whitening filter ${ }^{33,34}$ or changing the smoothing window length, ${ }^{35}$ in order to increase the number of states available from surface EMG signal. These techniques require a different muscle contraction for each controlled function, making the control of two or more joints very difficult. Other researchers have attempted to increase the information from one or two channels by using a time-series model. ${ }^{37.38}$ 


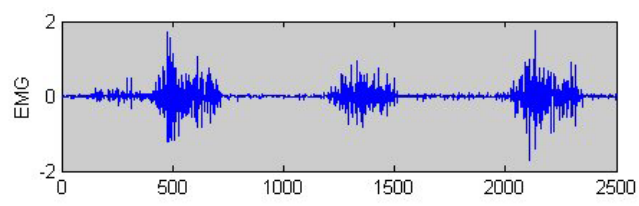

(a) Original EMG signal

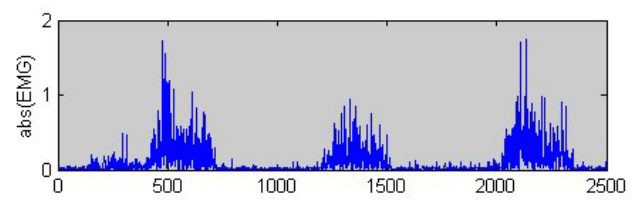

(b) Rectified EMG signal

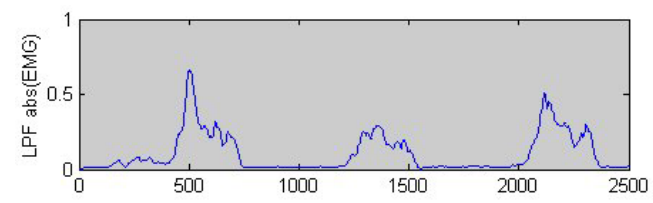

(c) Low pass filtered EMG signal

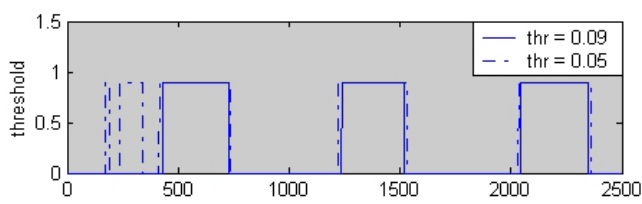

(d) Threshold-based detection of movement

FIGURE 3. Example of simple processing of EMG signal taken from biceps brachii.

Despite some promising results, this method turned out to be sensitive to changes in signal amplitude.

All these systems have been successfully implemented, but they cannot provide sufficient information to effectively control more than one DoF. Generally, all commercial myoelectric control systems are based on the common assumption that the instantaneous value of the myoelectric signal contains no information. Users are trained to produce a constant level of activation of muscles, and the prostheses are tuned according to these values. The steady-state EMG signal, however, has very little temporal structure because of the active modification 
of recruitment and firing patterns needed to sustain a contraction. ${ }^{32}$ The parameters that could be extracted to quantify its amplitude (e.g., variance, mean absolute value) or its frequency characteristics (e.g., Fourier spectrum, median frequency) are often not sufficient to distinguish between more than two classes of movement.

Starting from the 1990s, researchers found that there is useful information in the transient burst of myoelectric signal. Hudgins and colleagues ${ }^{39}$ showed that there is a considerable structure in the myoelectric signal during the onset of a contraction. Furthermore, this structure seemed to be different for different kinds of contraction, and further works ${ }^{40}$ demonstrated that transient EMG signals have a greater classification capacity than do steady-state signals. For this reason several features are extracted.

\section{Time Domain Features}

Features in the time domain are generally quickly calculated, because they do not need a transformation. Some or all of these features have been widely used in research and in clinical practice.

Mean absolute value (MAV) is an estimate of the mean absolute value of the signal $x_{i}$ in a segment $i$ that is $N$ samples in length. ${ }^{39,41,42}$

$$
\overline{X_{i}}=\frac{1}{N} \sum_{k=1}^{N}\left|x_{k}\right|, \quad \text { for } i=1, \ldots, I-1
$$

Sometimes ${ }^{43,44}$ the integrated absolute value $(\mathrm{IAV})=\mathrm{MAV} \cdot \mathrm{N}$ is used.

Mean absolute value slope (MAVSLP) $)^{39}$ is simply the difference between sums in adjacent segments $i$ and $i+1$ :

$$
\Delta \overline{X_{i}}=\overline{X_{i+1}}-\overline{X_{i}} \quad \text { for } i=1, \ldots, I-1
$$

Willison amplitude (WAMP $)^{45}$ is the number of counts for each change of the EMG signal amplitude that exceeds a predefined threshold. It is given by

$$
W A M P=\sum_{k=1}^{N} f\left(\left|x_{k}-x_{k+1}\right|\right)
$$

with $f(x)=1$ if $x>$ threshold, 0 otherwise. This unit is an indicator of firing of motor unit action potentials (MUAP) and, therefore, an indication of muscle contraction level. 
Variance of the EMG. Starting in late 1970s, the EMG signal was modeled as amplitude modulated Gaussian noise whose variance is related to the force developed by the muscle. ${ }^{32}$ The variance (or second-order moment) of the EMG is a measure of its power, and it is given by

$$
V A R=\sigma^{2}=\frac{1}{N-1} \sum_{k=1}^{N} x(k)^{2}
$$

Zero crossing $(\mathrm{ZC})^{39}$ is the number of times the waveform crosses zero. In order to reduce the noise-induced zero crossing, a threshold must be included. Given two consecutive samples $x_{k}$ and $x_{k+1}, \mathrm{ZC}$ is incremented if

$$
\operatorname{sgn}\left(-x_{k} \times x_{k+1}\right) \text { and }\left(\left|x_{k}-x_{k+1}\right| \geq \text { threshold }\right)
$$

with $\operatorname{sgn}(x)=1$, if $x>0,0$ otherwise. This parameter provides a rough estimate of the properties in frequency domain.

Slope sign changes $(\mathrm{SSC})^{14}$ is incremented if, given three consecutive samples $x_{k-1}, x_{k}$, and $x_{k+1}$

$$
\left(x_{k}-x_{k-1}\right) \times\left(x_{k}-x_{k+1}\right) \geq \text { threshold }
$$

for $k=1,1 / 4 \ldots, N$. This parameter provides an additional piece of information about frequency properties of the measured signal.

Waveform length $(\mathrm{WL})^{14}$ is the cumulative length of the waveform over the time segment. It is defined as

$$
l_{0}=\sum_{k=1}^{N}\left|\Delta x_{k}\right|
$$

where $D \Delta x_{k}=x_{k}-x_{k-1}$. This parameter gives a measure of waveform amplitude, frequency, and duration all in one.

Frequency ratio $(\mathrm{FR})$ was proposed in order to distinguish between contraction and relaxation of muscle in frequency domain. ${ }^{43}$ By applying the FFT to the EMG in time domain, the frequency ratio $\mathrm{FR}$ of the $-j^{\text {th }}$ channel is:

$$
F R_{j}=\frac{|F(\cdot)|_{j \text { low freq }}}{|F(\cdot)|_{j \text { high freq }}}
$$


The threshold for dividing low frequencies from high is decided through experiments. A high FR means that the degree of contraction of the muscle is high, a low FR means the opposite.

AR model. . $^{37,38,44,45}$ The EMG signal is nonstationary and nonlinear. But, in a short time interval, it can be regarded as a stationary Gaussian process. ${ }^{32}$ The EMG time series could be modeled as

$$
x_{k}=\sum_{i=1}^{n} a_{i} x_{k-1}+e_{k}
$$

where $n$ is the order of the AR model, $a_{i}$ are the estimate of the AR coefficients, and $e_{k}$ is the residual white noise. Graupe et al. ${ }^{37}$ first used the sequential least square (SLS) algorithm in order to minimize the difference $e_{k}$ between the estimate ${ }^{\wedge}\left(x_{k}\right)$ and the actual signal value $x_{k}$ at any sample time. A simple least square cost function can be formulated by taking the sum of squares of $e_{k}$ over a time window of the EMG, i.e.,

$$
\sum_{k=1}^{N}\left(\hat{x}_{k}-x_{k}\right)^{2}
$$

It can be shown ${ }^{46}$ that this minimization problem is reduced to a set of two recursive equations:

$$
\left\{\begin{array}{l}
\mathbf{a}_{k}=\mathbf{a}_{k-1}+\mathbf{P}_{k} \mathbf{X}_{k}\left(x_{k}-\mathbf{X}_{k}^{T} \mathbf{a}_{k-l}\right) \\
\mathbf{P}_{k}=\mathbf{P}_{k-1}-\frac{\mathbf{P}_{k-1} \mathbf{X}_{k} \mathbf{X}_{k}^{T} \mathbf{P}_{k-1}}{1+\mathbf{X}_{k}^{T} \mathbf{P}_{k-1} \mathbf{X}_{k}}
\end{array}\right.
$$

where $\mathbf{X}_{k}=\left(x_{k-1}, x_{k-2}, 1 / 4 \ldots, x_{k-p}\right), \mathbf{a}_{k}$ is the vector of the present estimates of the time series coefficients, $\mathbf{a}_{k-1}$ contains the previous estimates, and $\mathbf{P}_{k}$ is a $\mathrm{p}$ by $\mathrm{p}$ matrix. Initially $\mathbf{a}_{0}$ is set to zero and $\mathbf{P}_{0}$ is set to the identity matrix $\mathbf{I}$.

Other authors ${ }^{46,47}$ have used a Hopfield neural network in order to extract the AR coefficients. This method is faster than SLS or similar methods. The outputs $\hat{a}_{k}$ of the Hopfield network are calculated from the previous output weighted by the network weights $T_{k \mathrm{j}}$ and from the network inputs $I_{k}$ according to

$$
\hat{a}_{k(r+1)}=\hat{a}_{k r}+\lambda_{k}\left(\sum_{j=1}^{n} T_{k j} \hat{a}_{j r}+I_{k}\right)
$$

where $\hat{a}_{k r}$ is the $r^{\text {th }}$ estimate of $a_{k r}, \lambda_{k}$ is a gain parameter, and $n$ is the AR model order. The network converges to a value $(\hat{a})$ that minimizes the prediction error if 


$$
\left\{\begin{array}{c}
0<\lambda_{k}<\left(\frac{2}{\left|T_{k k}\right|}\right) \\
T_{k j}=-\sum_{l=n}^{M} y_{l-k} y_{l-j} \\
I_{j}=\sum_{l=n}^{M} y_{l-k} y_{k}
\end{array}\right.
$$

where $M$ is the number of data points considered.

Cepstrum Analysis. The Cepstrum of a signal is defined as the inverse Fourier transform of the logarithm of the magnitude of the power spectrum of the signal data. The system function $H(z)$ for the AR process in the $z$-domain is

$$
H(z)=\frac{1}{\sum_{l=1}^{n} a_{i} z^{-1}}
$$

$n$ being the order of the AR model and $a_{i}$ the AR coefficients. Then we can write

$$
\ln (H(z))=C(z)=\sum_{l=1}^{\infty} c_{i} z^{-1}
$$

Differentiating both sides with respect to $z^{-1}$ and equating the coefficients of like powers of $z^{-1}$, we derive the following recursive relation

$$
\begin{aligned}
c_{1} & =-a_{1} \\
c_{i} & =-a_{i}-\sum_{l=1}^{i-1}\left(1-\frac{l}{i}\right) a_{n} c_{i-l}
\end{aligned}
$$

for $1 \leq 1 \leq n$. For a transfer function with poles only, the first $P$ Cepstral coefficients can be obtained directly from the $P^{\text {th }}$ order coefficients of the AR model by using Eq. (16). In general, the use of cepstral method leads to a statistically significant improvement in the recognition rate respect to the use of AR method. An example of the use of cepstral coefficients is given in Park and Lee. ${ }^{48}$

\section{Time-Frequency Representation}

The fundamental purpose of feature extraction is to emphasize the important information in the measured signal while rejecting noise and irrelevant data. Time-frequency representation can localize the energy of the signal both in time and in frequency, thus allowing a more 
accurate description of the physical phenomenon. ${ }^{49}$ On the other hand, time-frequency representation (TFR) generally requires a transformation that could be computationally heavy.

Among all different types of TFR, discrete, linear TFRs - short -time Fourier transform (STFT), wavelet transform (WT), and wavelet packet transform (WPT)—are preferable to quadratic TFRs, which are too computationally intense for real-time application. The fundamental difference between linear TFRs is in the manner in which they partition the time-frequency plane (Fig. 4). The STFT has a fixed tiling; once specified, each cell has an identical aspect ratio. The tiling of the wavelet transform is variable - the aspect ratio of the cells varies such that the frequency resolution is proportional to the center frequency. This tiling has been shown to be more appropriate for many physical signals, but the partition is nonetheless still fixed. The WPT provides an adaptive tiling — an overcomplete set of tilings are provided as alternatives, and the best for a given application is selected.

Gabor transform or short-time Fouriertransform (STFT). Most transforms, in their original form, assume that the signal under consideration is stationary. Generally this assumption fails in the case of EMG signal, except for short periods of time. Given a finite-length sequence $x_{i}, i \in\{0, \ldots, L-1\}$, its discrete Fourier transform (DFT) is defined as

$$
X[m F] \equiv X[m]=\sum_{i=0}^{L-1} x[i] e^{-j 2 \pi(m F)\left(i T_{s}\right)}
$$

where $L$ is the length of the sequence, $F=1 / L T_{s}$ is the frequency sampling step size. The STFT consists of a series of DTFs, indexed with respect to $T_{s}$ and $F$

$$
\begin{aligned}
S T F T[k, m] & \equiv S T F T\left[k T_{s}, m F\right] \\
& =\sum_{i=1}^{L-1} x[i] g[i-k] e^{-j 2 \pi m i / L}
\end{aligned}
$$

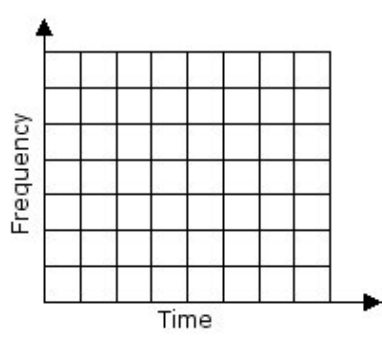

(a) STFT

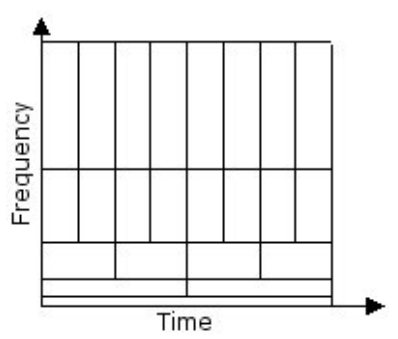

(b) WT

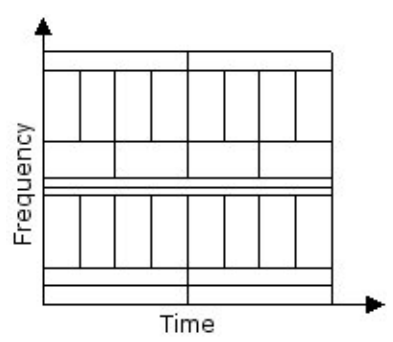

(c) WP

FIGURE 4. Different tiling of STFT, WT, and WPT. 


\section{CONTROL OF MULTIFUNCTIONAL PROSTHETIC HANDS}

where $g[i]$ is the window function. The temporal sampling step size is $T=K \cdot T_{s}$; if $K=1$, the STFT is computed at every sample in time; if $K=L$, the successive analysis windows do not overlap.

The resolution in time and frequency is lower bounded by the time-bandwidth uncertainty principle or Heisenberg inequality.

$$
\Delta t \cdot \Delta f \geq \frac{1}{4 \pi}
$$

A Gaussian window allows a balanced time resolution and frequency resolution.

The STFT has, among its other useful properties, a well-developed theory and can be computed very efficiently. ${ }^{39,50,51}$ The main constraint is that each cell in the time-frequency plane must have identical shape (Fig. 4a). In fact, as imposed by the temporal and frequency sampling steps, the time-frequency plane is divided into cells, each of which has a temporal width of $T$ and a frequency height of $F$, and clearly the energy distribution of physical signals is not (in general) conveniently localized in region of fixed aspect ratio.

Wavelet transform (WT). The WT overcomes the main drawback of the STFT by varying the time-frequency aspect ratio and by producing a good frequency resolution $\Delta f$ in long time windows (low frequencies) and a good time localization $\Delta t$ at high frequencies. ${ }^{49-54}$ This produces a tiling of the time-frequency plane that is appropriate for most physical signals (Fig. $4 \mathrm{~b})$.

The continuous wavelet transform is defined as

$$
C W T_{x}(\tau, a)=\frac{1}{\sqrt{a}} \int x(t) \Psi\left(\frac{t-\tau}{a}\right) d t
$$

where $\Psi(t)$ is the mother wavelet, which has the property that the set $\left\{\Psi_{a, b}\right\}$ forms an orthonormal basis in $L^{2}(\Re)(a, b \in \mathfrak{R}, a \neq 0)$.

In its discrete form, $a=a_{0}{ }^{j}$ and $\tau=n \cdot a_{0-j}$ where $n$ and $j$ are integers (discrete wavelet transform, or DWT). A common choice is $a_{0}=2$ (dyadic wavelet basis), which allows great computational efficiency.

Wavelet Packet Transform (WPT). WPT is a generalized version of the CWT and the DWT. Because the CWT is redundant, the tiling of the time-frequency plane is configurable (Fig. 5). The basis for the WPT is chosen using an entropy-based cost function. ${ }^{55}$ While the WT is extremely computationally efficient (it takes less than $2 \mathrm{~ms}$ to calculate the WT of a 256-ms stream of data), the WPT demands substantially greater computation, about $200 \mathrm{~ms}$ on a record length of $256 \mathrm{~ms}$. Both WT and WPT have been tested on EMG signals by Englehart and colleagues, ${ }^{40,49,56}$ showing that time-frequency representation, together with a linear dimensionality reduction, is capable of a better description of the intended movement. For a detailed mathematical description of WT and WPT, see Vetterli and Kovacević. ${ }^{54}$ 


\section{II.D Dimensionality Reduction}

The reduction of the dimensionality of the problem is generally fundamental to increasing the classification performance. This process should preserve as much of the relevant information as possible while reducing the number of dimensions.

The two main strategies of dimensionality reduction are feature selection and feature projection. ${ }^{49}$

\section{Feature Projection}

This method, instead of searching the best subset of features, tries to determine the best combination of the original features to form a new feature set, generally smaller than the original one. Moreover, if the map $f: V \rightarrow Z$ is linear, finding the coefficients of this projection could be quite a fast and simple process.

Principal component analysis, or PCA, also knows as Karbunen-Loéve transform or singular value decomposition (SVD), provides a linear map from the original set of variables $v \in V \subseteq \mathfrak{R}^{\mathrm{M}}$ into a reduced-dimension set of uncorrelated variables $z \in Z \subseteq \mathfrak{R}^{\mathrm{L}}$ (the principal components), minimizing the mean-square error (MSE) between the original feature set and the projected one. ${ }^{40,57}$ The transformed variables are ranked according to their variance, thereby reflecting a decreasing effectiveness in representing the original set of variables.

This technique is effective in pattern recognition, reducing the complexity of the resulting feature space and eliminating linear dependencies among data. The minimum error is reached when the original feature set is projected onto the orthonormal basis $\mathbf{u}_{i}$ so that $\sum_{v} \mathbf{u}_{i}=\lambda_{i} \mathbf{u}_{i} ; \mathbf{u}_{i}$ are the eigenvectors and $\lambda_{i}$ the eigenvalues of $\sum_{v}$ (the covariance matrix of the original set of vectors $\mathbf{v}$ ).

Sometimes there are features that are best represented by a nonlinear combination of the original variables. Multilayer neural networks ${ }^{58}$ or self-organizing maps, ${ }^{59}$ in these cases, can obtain a successful nonlinear dimensionality reduction. This method is computationally heavy, and thus generally PCA or feature selection methods are preferred.

\section{Feature Selection}

In this case, the best subset $\mathbf{z} \in \mathrm{Z} \subseteq \mathfrak{R}^{\mathrm{L}}$ of the original feature set $\mathbf{v} \in \mathrm{V} \subseteq \mathfrak{R}^{\mathrm{M}}$ is chosen according to some criteria for judging whether one subset is better than another. The ideal criterion for classification should be the minimization of the probability of misclassification, but generally simpler criteria based on class separability are chosen. Furthermore, the exhaustive search among all possible subsets is often impractical, and some nonexhaustive and sequential methods are used.

In general, feature selection methods use class membership to determine discriminant power. As the original identity of the features is maintained, the utility of each individual feature is known. On the other hand, some of the original features are completely discarded, and all the information they convey is completely lost. Feature selection methods behave well on well-structured and poorly redundant data.

An example of feature selection is shown in Micera et al. ${ }^{57}$ In order to reduce the number of muscles to those truly relevant to the movement under investigation, the coefficient of 
expressiveness $(\mathrm{CoE})$ was introduced. For the $l^{\text {th }}$ muscle and the $r^{\text {th }}$ movement the following expression was evaluated:

$$
k_{j, l, r}=\sum_{i=1}^{n}\left|c_{i, l, r}\right| \frac{\lambda_{i}}{\sum_{m=1}^{p} \lambda_{m}} \quad j=1, \ldots, J
$$

where $n$ is the number of principal components (PCs) that are "expressive", $c_{i, l, r}$ is the correlation coefficient between the $i^{\text {th }} \mathrm{PC}$ and the selected muscle for the $r^{\text {th }}$ movement, $\lambda_{i}$ is the eigenvalue associated to the $i^{\text {th }} \mathrm{PC}, p$ is the total number of eigenvalues, and $J$ is the number of trials. Then

$$
K_{l, r}=\sum_{j=1}^{J} k_{j, l, r}
$$

and, for the $r^{\text {th }}$ movement, we normalize the $K_{l, r}$ coefficients by their maximum value among the selected muscles, yielding the $\mathrm{CoE}$.

\section{II.E. EMG Pattern Classification}

There are several possible classification techniques. ${ }^{60}$ Among them, the most used are Bayesian pattern classifiers and artificial neural networks. Recently, some authors have tried to use a fuzzy classifier, but other authors reported that with the appropriate representation of the signal, a linear classifier performs better than a nonlinear one. ${ }^{40,56}$

\section{Bayesian Pattern Classification}

One of the standard statistical classification methods is the Bayes classifier. The measurement of the input vector $\mathbf{x} \in \mathrm{X} \subseteq \mathfrak{R}^{\mathrm{N}}$ and of its response space $y \in \mathrm{Y}=\left\{y_{1}, \ldots, y_{K}\right\}$ may be considered in a probabilistic framework, and viewed as single observation of the random variables $X$ and $Y$. The a posteriori probability that pattern $\mathbf{x}$ comes from class $y_{k}$ is given by

$$
P\left(y_{k} \mid X\right) \text { for } k=1, \ldots, K
$$

These probabilities, in general, are not known but may be calculated from the a priori probabilities $P\left(y_{k}\right)$ and the conditional density function $p\left(x \mid y_{k}\right)$ using the Bayes' theorem:

$$
P\left(\mathbf{x}, y_{k}\right)=P\left(y_{k}\right) p\left(\mathbf{x} \mid y_{k}\right)=p(\mathbf{x}) P\left(y_{k} \mid \mathbf{x}\right)
$$

where 


$$
p(\mathbf{x})=\sum_{j=1}^{K} P\left(y_{j}\right) p\left(\mathbf{x} \mid y_{j}\right)
$$

Note that $p(\mathbf{x})$ is the probability density function of the input space, and it remains constant for all $P\left(y_{k} \mid X\right)$. Bayes' decision rule minimizes the probability of classification error.

If it is possible to make some assumption about the form of the conditional probability density functions $p\left(\mathbf{x} \mid y_{j}\right)$, then the Bayes classifier could be dramatically simplified. ${ }^{49,60}$

\section{Artificial Neural Networks}

An artificial neural network $(\mathrm{ANN})^{47}$ is a computational system inspired by the learning characteristics and structure of biological neural networks. Graupe et al. ${ }^{38}$ showed that conventional pattern recognition techniques can be successfully used for classifying single-site ME signals, achieving a good discrimination among signals, but only after hours of subject training. The application of ANN could reduce the amount of user training. Moreover, a simple feed-forward neural network can assure high recognition rates.

ANNs possess several attractive features that make them suitable for difficult signal processing problems: generalization and ability to learn from experience without requiring an a priori mathematical model of the underlying signal characteristics; adaptation to changing environmental conditions; and the ability to process degraded and incomplete data. ${ }^{61-63}$ However, they have the problem of learning from a limited number of examples. ${ }^{47}$

One of the simplest and most widely used ANN is the multilayer perceptron (MPLP), which is characterized by a set of input units, a layer of output units, and a number of hidden layers (one or two, in general). Each input node is connected to each unit in the hidden layer. The connections between units have an associated weight $W$; each unit of the hidden layer is connected to the neurons in the following layer, be it hidden or output, in a similar way. The input units retain the analog value of each input. Hidden and output nodes, however, have a transfer function $F(\alpha)$, where $\alpha=\sum_{i=1}{ }^{n} w_{i} a_{i}$ is the total input of the neuron, $a_{i}$ is the $i^{\text {th }}$ input, and $w_{i}$ is its weight.

The original perceptron conceived by Rosenblatt in 1958 used a hard-limiting nonlinearity:

$$
f_{H L}(s)=\left\{\begin{array}{l}
1, s>0 \\
0, s \leqslant 0
\end{array}\right.
$$

Other commonly used functions are the logistic sigmoid nonlinearity, which is differentiable,

$$
f_{\log }(s)=\frac{1}{1+e^{-\beta s}}
$$

and the hyperbolic tangent sigmoid, whose output ranges from -1 to +1 : 


$$
f_{\tanh }(s)=\frac{e^{\beta s}-e^{-\beta s}}{e^{\beta s}+e^{-\beta s}}
$$

Many algorithms have been developed to train the network-i.e., to adapt the network weights. These algorithms can be divided into supervised, in which the desired response is available during the learning phase, and unsupervised, in which it is not. Generally, the connection weights and neuron threshold values are varied by using the back propagation (BP) algorithm..$^{47,60}$

\section{Fuzzy Logic}

Fuzzy inference systems (FISs), like ANNs, are excellent at developing human-made systems that can perform information processing in a manner similar to our brain. ${ }^{64}$ Fuzzy logic systems can emulate human decision-making more closely than ANNs, because of the possibility of introducing the knowledge of an expert in the fuzzy rules in the form IF-THEN. Fuzzy systems can be broadly categorized into two families. The first includes linguistic models bases on the collection of IF-THEN rules, whose antecedents and consequents use fuzzy values. An example of this group is the Mamdani model. The knowledge is represented as

$$
\begin{aligned}
& R^{i}=\text { If } x_{1} \text { is } A_{1}^{i} \text { and } x_{2} \text { is } A_{2}^{i} \ldots \text { and } x_{n} \text { is } A_{n}^{i} \\
& \text { then } y^{i} \text { is } B^{i}
\end{aligned}
$$

where $R^{i}(I=1,2, \ldots, l)$ denotes the $I^{\text {th }}$ fuzzy rule, $x_{j}(j=1,2, \ldots, n)$ is the $n^{\text {th }}$ input, $y^{i}$ is the output of the fuzzy rule $R^{i}$, and $A_{1}{ }^{i}, A_{2}{ }^{i}, \ldots, A_{m}{ }^{i}, B^{i}(I=1,2, \ldots, l)$ are fuzzy membership functions. ${ }^{65}$ The second category is based on the Tagagi-Sugeno-type systems and uses a rule structure that has fuzzy antecedent and functional consequent parts:

$$
\begin{aligned}
& R^{i}=\text { If } x_{1} \text { is } A_{1}^{i} \text { and } x_{2} \text { is } A_{2}^{i} \ldots \text { and } x_{n} \text { is } A_{n}^{i} \\
& \text { then } y^{i}=a_{0}^{i}+a_{1}^{i} x_{1}+\ldots+a_{n}^{i} x_{n}
\end{aligned}
$$

This approach approximates a nonlinear system with a combination of several linear systems by decomposing the whole input space into several partial fuzzy spaces and representing each output space with a linear equation. If one is interested in a more precise solution (and is not so interested in a precise linguistic interpretability) Sugeno-type systems are more suitable; on the other hand, Mamdami-type systems are best suited to obtain a good linguistic interpretability. Some examples of fuzzy systems applied to EMG classification are given in the literature. ${ }^{42,43,57,66}$

\section{Neuro-Fuzzy Systems}

Fuzzy logic and neural systems have very contrasting application requirements. Fuzzy systems, one the one hand, are more appropriate when sufficient expert knowledge about the process 


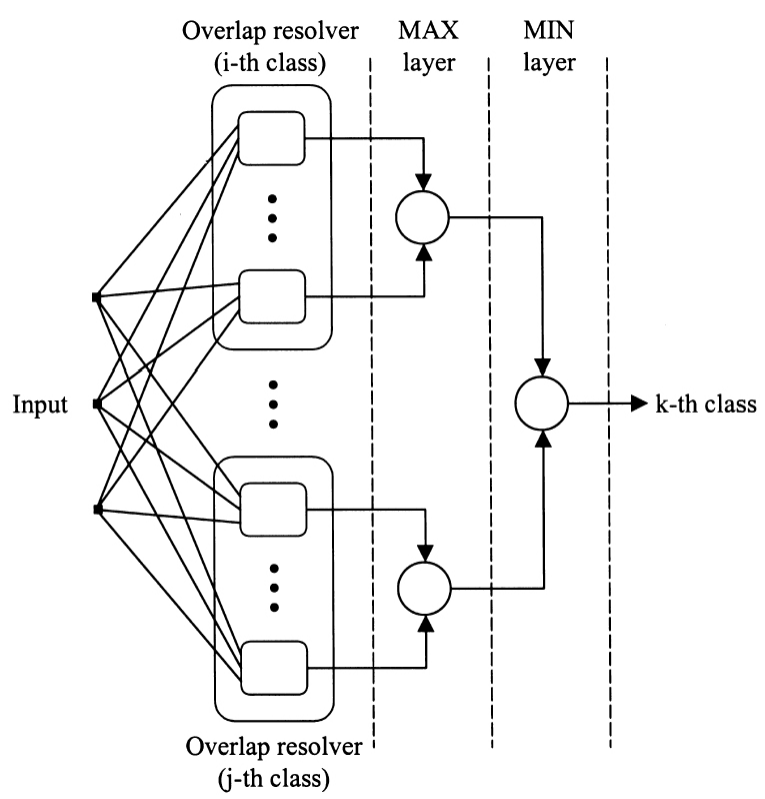

FIGURE 5. Architecture of Abe-Lan fuzzy classifier. (Reprinted from Micera et al. ${ }^{57}$ with permission.)

is available; neural networks, on the other, are more useful when sufficient data are available or measurable. Both these approaches build nonlinear systems, either numerical (ANNs) or symbolic (FIS). However, fuzzy systems exhibit both symbolic and numeric features. Therefore, the integration of neural and fuzzy systems leads to a "symbiotic" relationship in which fuzzy systems provide a powerful framework for expert knowledge representation, while neural networks provide learning capabilities and exceptional suitability for computationally efficient hardware implementations. ${ }^{65,67}$

Neuro-fuzzy computing enables to us build more intelligent decision-making systems by combining the advantages of ANNs (massive parallelism, robustness, learning in a data-rich environment) with the fuzzy modeling of imprecise and qualitative knowledge.

An example of neuro-fuzzy classifier is the Abe-Lan network. ${ }^{57,68}$ The input space is subdivided into a number of activation hyperboxes, corresponding to regions in the feature space that contain all the training data available for each class. Hyperboxes may overlap, and in this case an inhibition hyperbox (corresponding to the overlapping region) is defined. Recursively, a number of activation and inhibition hyperboxes at progressively higher levels can be defined for each class pair, until all overlaps are resolved. In the rule set, a corresponding fuzzy rule is associated to each hyperbox, be it activation or inhibition (Fig. 5).

The second-layer units consist of fuzzy rules that calculate the degrees of membership for the rules that resolve the overlaps between activation hyperboxes. The third-layer units for the $I^{\text {th }}$ class take the maximum value of inputs from the second layer; the fourth-layer units take the minimum value among the maximum values generated by the third layer. Finally, an unknown feature vector is classified as the class with the maximum membership assignments.

Results $^{57}$ showed that the proposed classifier is able to correctly identify all the EMG signals related to the different movements. 


\section{II.F Offline and Online Learning}

EMG signal patterns differ among individuals. Moreover, electrical impedance of the skin; electrode locations; time variations caused by fatigue, sweat, and so on differ from user to user and from time to time. It is clear that the EMG processing unit should adapt itself to these changes in order to minimize ill-discriminations. The device should "learn" how the user behaves and adjust its internal parameters relative to the operator's variation in real time.

Most current prostheses, however, are tuned only in the offline phase. The user learns to reproduce one or two different signals, and the prosthesis is tuned to these signals. When the user cannot control the prosthesis properly, she/he should come back to the assistance center and retune the controller. It is easy to understand that with such a controller it is not possible to successfully control more than one active DoF, because the differences between tuned signals and actual ones tend to increase gradually with time.

Nishikawa and his group ${ }^{69-71}$ proposed a real-time learning method that makes it possible to control up to ten different motions of the forearm ${ }^{69}$ from two channels of EMG with a success rate of up to $91.5 \%$. The controller is composed of three modules: the analysis unit, which generates a feature vector containing useful information for discriminating motions from measured EMG signal; the adaptation unit, which makes a mapping function from the feature vector; and the trainer unit, which generates training data from the teacher signal sent by the operator and the feature vector at the moment. When the trainer unit receives teacher signal $T$ from the operator, it generates the teaching vector $\mathbf{d}$ by formulæ

$$
\begin{aligned}
\mathbf{d} & =\left(d_{1}, d_{2}, \ldots, d_{n_{m}}\right) \\
d_{i} & = \begin{cases}0.9 & \text { if } i=T \\
0.1 & \text { otherwise }\end{cases}
\end{aligned}
$$

where $n_{m}$ is the total number of the cells in the $m^{\text {th }}$ layer of the feed-forward neural network of the adaptation unit.

In the system proposed by Fukuda, ${ }^{72}$ the EMG pattern vectors $\mathbf{x}(n)$ for forearm motions of the operator are measured during motions and are used for offline learning. The entropy $H(s)=-\sum_{k=1}{ }^{K} Y_{k}(n) \log _{2} Y_{k}(n)$ of the output of the neural network is compared with the threshold of the online learning $\theta_{0}$; if it is lower, a pair of $\mathbf{x}(n)$ and the output motion is added to the set of learning data, and the oldest stored set is deleted. The entropy is also used for a motion suspension rule, because if it is over the determination threshold $\theta_{d}$, it could indicate that the network output is ambiguous (for a detailed explanation of this method the reader is referred to Fukuda et al. ${ }^{72}$ ).

\section{CONTROL OF MULTIFUNCTIONAL PROSTHETIC HANDS USING EMG}

The main objective of this article is to analyze critically different methods for EMG signal processing that could be exploited to improve the controllability and thus the usability of current hand prostheses. The motivation for this effort is the assumption that, even if current hand prostheses have many intrinsic limitations, their performance could be 


\section{ZECCA ET AL.}

improved significantly by implementing better control techniques based on better EMG signal processing.

Replicating the performance of the human hand is beyond current technical capabilities. In fact, the human hand is extremely complex: it has $22 \mathrm{DoFs}$, controlled by about 38 muscles in the hand (almost twice the number of $\mathrm{DoFs}^{3,73}$ ), and it incorporates about 17,000 tactile units of 4 different types ${ }^{74,75}$ with different receptive fields and different sensitivity to static and dynamic events.

Commercial hand prostheses have a limited number of DoFs (one or two for finger movements and thumb opposition), and thus they have low grasping functionality. In fact, they do not allow adequate encirclement of objects, compared to the adaptability of the human hand. ${ }^{8}$ Moreover, their low compliance leads to instability of the object in the presence of external perturbations. ${ }^{76}$ The main advantage of current prosthetic hand devices is that they can generate large grasping forces $(>100 \mathrm{~N})^{77}$ and are simple to implement and control, in particular by using EMG signal.

During the last two decades several robotic and anthropomorphic hands have been developed. All these hands have a high number of DoFs (up to 16), and a dexterity comparable to that of the human hand. Some examples of robotic hands are the Utah/MIT hand, ${ }^{78}$ the Stanford/JPL hand, ${ }^{79}$ the DLR hand, ${ }^{80}$ and the Robonaut Hand ${ }^{81}$ Unfortunately, none of these hands can be used as prostheses, because their actuation and control systems are quite heavy and bulky, and thus they cannot be embedded within the hand. Table 1 presents a comparison among the human hand, some hand prostheses, and some robotic hands.

A design solution that could improve the dexterity of a prosthesis while maintaining intrinsic actuation (i.e., all the actuators embedded within the hand structure) is based on underactuated mechanisms (i.e., a mechanism that has fewer actuators than degrees of freedom ${ }^{13,82}$ ). Underactuated mechanisms allow grasping objects in a way that is closer to human grasping than independent actuation, but their main limitation is the control of this functionality.

Electromyographic signal is a simple and easily obtained source of information on what the users of a prosthesis would like to do with their artificial hands. Surface electrodes are easy to use and manage, and they do not require surgery. Moreover, there are no harnesses

Table 1. Comparison of Human Hand, Hand Prostheses, and Robotic Handsa

\begin{tabular}{|c|c|c|c|c|c|c|}
\hline & $\begin{array}{l}\text { \# of } \\
\text { DoFs }\end{array}$ & $\begin{array}{l}\text { Size of the hand } \\
\text { (normalized) }\end{array}$ & $\begin{array}{c}\# \text { of } \\
\text { Fingers }\end{array}$ & $\begin{array}{c}\text { \# of } \\
\text { Sensors }\end{array}$ & $\begin{array}{c}\text { \# of } \\
\text { Actuators }\end{array}$ & $\begin{array}{c}\text { Opposable } \\
\text { Thumb }\end{array}$ \\
\hline Human Hand ${ }^{3}$ & 22 & 1 & 5 & $17^{\prime} 000$ & 38 & yes \\
\hline MARCUS Hand 5 & 2 & 1.1 & 3 & 5 & 2 & no \\
\hline Ottobock/SUVA Hand ${ }^{7}$ & $1+1$ & 1 & 3 & 1 & 1 & no \\
\hline Utah/MIT Hand78 & 16 & $2+$ control & 4 & & 32 & yes \\
\hline Stanford/JPL Hand ${ }^{79}$ & 9 & $1.2+$ control & 3 & & 12 & no \\
\hline DLR Hand $\|^{80}$ & 13 & $1.5+$ control & 4 & 64 & 13 & no \\
\hline Robonaut Hand 81 & $12+2$ & $\begin{array}{c}\text { Astronaut } \\
\text { gloved hand + } \\
\text { control }\end{array}$ & 5 & $\begin{array}{c}43+ \\
\text { tactile }\end{array}$ & 14 & yes \\
\hline
\end{tabular}

a The size of the artificial hands is normalized with respect to the size of the human hand 


\section{CONTROL OF MULTIFUNCTIONAL PROSTHETIC HANDS}

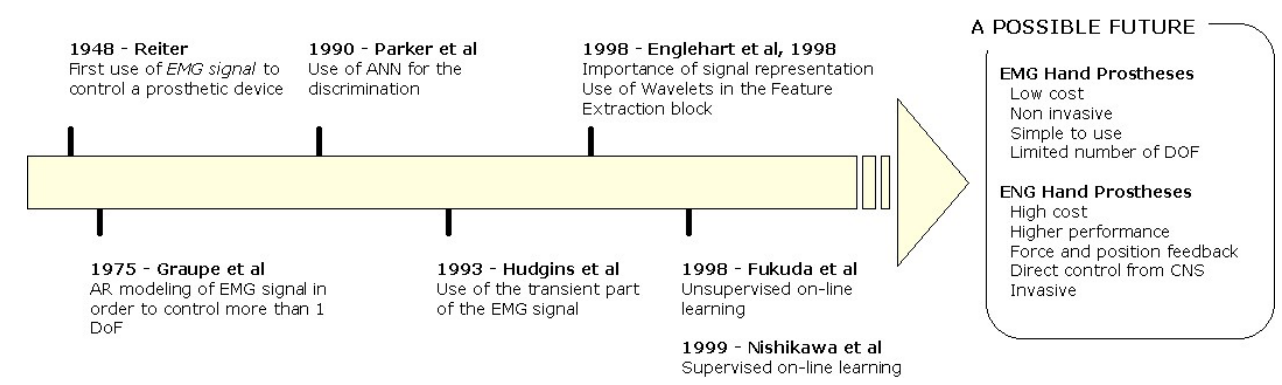

FIGURE 6. Evolution of use of EMG signal to actively control prosthetic hand.

that could limit the movement of the forearm. It is possible to control an active device with just one differential electrode placed on the residual limb, even in infants. ${ }^{89}$ The technology of EMG signal processing is making steady progress. The evolution of the use of the EMG signal to actively control a prosthetic hand is showed in Figure 6.

Reiter, ${ }^{21}$ in 1948 , was the first to use the EMG signal to control a simple prosthetic device. Nowadays, all prosthetic devices used in clinical practice have one ore two active DoFs, directly controlled by a couple of electrodes placed on two antagonist muscles, either in proportional or on/off mode..$^{22}$ The use of a larger number of electrodes to control more active DoFs has several drawbacks, because the coding of movements and the number of electrodes would greatly increment the problems in fabricating and using the socket.

Starting in 1975, some research groups ${ }^{36-38}$ realized that a correct modeling of the EMG signal could make it possible to control a device with more than just one DoF. Unfortunately, the hardware and software resources available at that time were not sufficient to realize a device that could be used in clinical practice. In particular, Graupe et al., ${ }^{38}$ with AR modeling and Bayesian discrimination, were able to successfully discriminate among six different classes of movement, with a success rate of up to $99 \%$. Unfortunately, these results were obtained only after 12 hours of user training, and performance significantly degraded with time, because of the modification of EMG generated by the user. The use of $\mathrm{ANNs}^{84}$ could reduce the time needed for the user training, but the problems of the modification related to signal variation with time and from person to person still remain.

In all these works EMG signal was used in its stationary phase, and users were trained to contract their residual muscles in order to obtain constant levels of EMG. In this way the control of the prosthetic device was simplified (it could be a simple proportional control, as showed in Dorcas and $\mathrm{Scott}^{85}$ ) but a lot of information was ignored.

In 1993 Hudgins et al..$^{39}$ first proposed a new control strategy for artificial devices. In fact, they observed that there is considerable structure in the myoelectric signal during the onset of a contraction. This structure is distinct for different limb movements and could be used as source of information for classifying the EMG signal. They were able to discriminate between four different movements with just one bipolar electrode by extracting MAV, MAVSLP, ZC, SSC, and WL from the measured signal, with a two-layer ANN. However, the discrimination error was still quite high $(>10 \%)$.

In recent years, EMG signal has been largely investigated both for the realization of multifunctional myoelectric prostheses ${ }^{40,61,71}$ and for the improvement of teleoperation of robotic devices, ${ }^{14}$ but as yet all these systems are not capable of successfully controlling a multifunctional hand. The major problem is the time-variant characteristics of the EMG 
signal, due to physiological changes in the muscles and to the changes in the coupling between skin and the electrodes. An equally important problem is the stochastic nature of the EMG, resulting in parameter estimation errors that, in turn, cause classification and/or control difficulties. Moreover, some control errors are generally introduced by the inability of the patient to reliably generate and reproduce the target contraction signals (operator errors). A possible solution is the realization of an online learning module, either supervised ${ }^{70,71}$ or unsupervised, ${ }^{72}$ to continuously adapt the parameters of the classifier. For example, Nishikawa and colleagues ${ }^{69}$ were able to discriminate among ten different movements of the forearm on three normal subjects with two-channel EMG signals by using Gabor Transform, MAV, and feed-forward ANN, but the success rate was lower than $90 \%$.

Some of the problems of myoelectric control could be overcome by automating some grasping functions. ${ }^{5,86,87}$ Some attempts to control multifunctional devices by using more than two electrodes ${ }^{88}$ have been made, and the use of nerve-muscle grafts ${ }^{89}$ have been proposed, but increasing the number of electrodes is not useful in clinical practice, because it introduces additional discomfort in using the prosthesis.

\section{CONCLUSIONS}

In the last thirty years, many research efforts have been carried out in the myoelectric control field. Several techniques have been developed to control multifunctional prosthetic devices, and many of them showed promising results. Moreover, these techniques could be also applied in other fields, not only in the control of myoelectric prostheses. For example, algorithms for detecting the activation of muscles are quite useful in gait analysis. ${ }^{90}$ However, despite all these efforts, EMG signal analysis seems to be quite limited in the number of possible functions that can be restored by using a few electrodes. Moreover, the EMG signal cannot provide any feedback to the user.

A possible solution to overcome the limits of the EMG-based approach could be the realization of an interface between the peripheral nervous system (PNS) and the artificial device (i.e., a "natural" neural interface [NI]) to record and stimulate the PNS in a selective way. ${ }^{91-94}$ Recent developments in the technology of electronic implants and in the understanding of nerve functions have made it possible to fabricate selective neural interfaces that work by interchanging information between the nervous system and computerized artificial instruments. A biocompatible neural interface can restore some sensory feedback to the user by stimulating in an appropriate way the afferent nerves and can allow motor control of the prosthesis based on a "natural" ENG-based control. This will be possible by focusing appropriate research efforts on the technological development of the neural interface and on the characterization of the PNS afferent signals in response to mechanical and proprioceptive stimuli. When the patient receives sensory feedback from the stimulation of the afferent nerves, and the prosthetic device is controlled directly through the efferent nerves, the user will again be able to "feel" the hand as part of the body.

In conclusion, with these considerations in mind, two solutions for controlling hand prostheses could be envisaged. On the one hand, EMG-controlled prostheses could represent a "cheap" solution (i.e., low cost and noninvasive) for the restoration (even if partial) of some hand functions. On the other hand, a multifunctional "cybernetic" hand prosthesis with ENG-based control would be a more sophisticated solution.

It is worth noting that this situation is already present in the field of neuroprostheses, 
where we can find the "noninvasive" solution - e.g., the Handmaster system ${ }^{95}$ which comprises a hand-forearm orthosis containing an array of electrodes connected to a portable electronic microprocessor-controlled unit, and which is designed for simple and independent positioning by the patient; and the "invasive" solution-e.g., the Freehand system, ${ }^{98}$ which consists of a pacemaker-like stimulator implanted in the chest, which sends electrical impulses from an external control/power source through lead wires to eight electrodes implanted in the muscles of the forearm and hand.

\section{ACKNOWLEDGMENTS}

This work has been partially supported by a research project entitled Design and Development of Innovative Components for Sensorized Prosthetic Systems, currently ongoing at the Applied Research Center on Rehabilitation Engineering of Viareggio, Italy, funded by National Institute for Insurance of Injured Workers (INAIL), and originated by a joint initiative promoted by INAIL and Scuola Superiore Sant'Anna. This work is supported in part also by funds of the CYBERHAND (Development of a Cybernetic Hand) Project, IST-FET Project \#2001-3509.

\section{REFERENCES}

1. Chao E, An K, Cooney III W, Linschied R. Biomechanics of the Hand. Singapore: World Scientific, 1989.

2. Kapandji IA. The Physiology of the Joints. Vol. 1, Upper Limb. Edinburgh: Churchill Livingstone, 1982.

3. Kandel E, Schwartz J, Jessel T. Principles of Neural Science. 3 ed. New York: Elsevier Science, 1985.

4. Cupo ME, Sheredos SJ. Clinical evaluation of a new, above elbow, body powered prosthetic arm: a final report. J Rehab Res Dev 1998; 35:431-444.

5. Kyberd PJ,Holland OE, Chappel PH, Smith S, Tregidgoi R, Bagwell PJ, Snaith M. MARCUS: A two degree of freedom hand prosthesis with hierarchical grip control. IEEE Trans Rehab Eng 1995; 3:70-76.

6. Agnew P. Functional effectiveness of a myoelectric prosthesis compared with a functional split hook prosthesis: A single subject experiment. Prosthet Orthot Int 1981; 5:92-96.

7. Otto Bock HealthCare GmbH, http://www.ottobock.com. Duderstadt (DE), 2002.

8. Cutkosky MR. Robotic Grasping and Fine Manipulation. Boston: Kluwer Academic, 1985.

9. Carrozza M, Micera S, Massa B, Zecca M, Lazzarini R, Canelli N, Dario P. The development of a novel biomechatronic hand-ongoing research and preliminary results. Advanced Intelligent Mechatronics AIM 2001, Como, Italy, July 8-11 2001; 249-254.

10. Carrozza M, Dario P, Lazzarini R, Massa B, Zecca M, Roccella S, Sacchetti R. An actuating system for a novel biomechatronic hand. Proc. of the 7th Intl Conf on New Actuators, Actuator 2000, Bremen, Germany, June 19-20 2000; 276-280.

11. Carrozza M, Massa B, Dario P, Lazzarini R, Zecca M, Micera S, Pastacaldi P. A two DOF finger for a biomechatronic artificial hand. Technol Health Care 2002; 10:77-89.

12. Hirose S, Umetami Y. The development of soft gripper for the versatile robot hand. Mech Machine Theory 1977; 13:351-359.

13. Massa B, Roccella S, Carrozza MC, Dario P. Design and development of an underactuated 


\section{ZECCA ET AL.}

prosthetic hand. Intl Conf on Robotics and Automation (ICRA) 2002, Washington DC, May 11-15 2002; 3374-3379. IEEE, 2002.

14. Farry KA, Walker ID, Baraniuk RG. Myoelectric teleoperation of a complex roboitic hand. IEEE Trans Rob Autom 1996; 12:775-788.

15. Craelius W, Abboudi RL, Newby NA. Control of a muli-finger prosthetic hand. ICORR'99: Int Conf Rehab Rob, Stanford University, Stanford, California, July 1-2 1999; 255-260.

16. Curcie DJ, Flint JA, Craelius W. Biomimetic finger control by filtering of distributed forelimb pressures. IEEE Trans Neural Syst Rehabil Eng 2001; 9:69-75.

17. Kenney L, Lisitsa I, Bowker P, Heath G, Howard D. Dimensional change in muscle as a control signal for powered upper limb prostheses: A pilot study. Med Eng Physics 1999; 21: 589-597.

18. Weir R, Heckathorne C, Childress DS. Cineplasty as a control input for externally powered prosthetic components. J Rehabil Res Dev 2001; 38:357-363.

19. Childress DS, Weir RF. Quantitative assessment of direct muscle attachment to act as a control input for externally powered prostheses. Proc. 8th World Cong Intl Soc Prosthetics Orthotics, Melbourne, Australia, April 2-7 1995; 101.

20. Simpson DC. The choice of control system for multimovement prostheses: extended physiological proprioception (EPP). In: Herberts P, et al., editor. The Control of Upper-Extremity Prostheses and Orthoses. Springfield, IL: Charles Thomas, 1975; 146-150.

21. Reiter R. Eine neue elecktrokunsthand. Grenzgebiete der Medizin 1948; 4:183.

22. Scott RN, Parker PA. Myoelectric prostheses: state of the art. J Med Eng Technol 1988; 12: 143-151.

23. Heger H, Millstein S, Hunter GA. Electrically powered prostheses for the adult with an upper limb amputation. J Bone Joint Surg 1985; 67B:278-281.

24. Meier RH, Atkins DJ. Comprehensive Management of the Upper Limb Amputee. New York: Springer-Verlag, 1989.

25. Miguelez JM. Critical factors in electrically powered upper extremity prosthetics. J Proc Am Acad Orthot Prosthet Online (AAOP) 1999; 14:36-38.

26. Staude G, Wolf W. Objective motor response onset detection in surface myoelectric signals. Med Eng Phys 1999; 21:449-467.

27. Micera S, Sabatini AM, Dario P. An algorithm for detecting the onset of muscle contraction by EMG signal processing. Med Eng Phys 1998; 20:211-215.

28. Hermens HJ, Freriks B, Merletti R, Stageman D, Blok J, Rau G, Disselhorst-Klug C, Hägg G, editors. SENIAM (Deliverable 8). European Recommendations for Surface ElectroMyoGraphy, Roessingh Research and Development b. v. 1999. Program: BIOMED-2, 1996/98.

29. De Luca CJ, Surface electromyography: detection and recording. 1996. http://www.delsys.com/ library/papers/SEMGintro.pdf.

30. Staude G, Flachenecker C, Daumer M, WolfW. Onset detection in surface electromyographic signals: a systematic comparison of methods. EURASIP J Appl Signal Proc 2001; 2:67-81.

31. Micera S, Vannozzi G, Sabatini AM, Dario P. Improving detection of muscle activation intervals. IEEE Eng Med Biol Mag 2001; 20:38-46.

32. Bashamajian JV, De Luca CJ. Muscles Alive. Baltimore, MD: Williams \&Wilkins, 1985.

33. Clancy EA, Farry KA. Adaptive whitening of the electromyogram to improve amplitude estimation. IEEE Trans Biomed Eng 2000; 47:709-719.

34. Park E, Meek SG. Adaptive filtering of the electromyographic signal for prosthetic control and force estimation. IEEE Trans Biomed Eng 1995; 42:1048-1052.

35. St-Amant Y, Rancourt D, Clancy EA. Influence of smoothing window lenght on electromyogram amplitude estimates. IEEE Trans Biomed Eng 1998; 45:795-799.

36. Saridis GN, Gootee TP. EMG pattern analysis and classification for a prosthetic arm. IEEE Trans Biomed Eng 1982; 29:403-412.

37. Graupe D, Cline WK. Functional separation of EMG signals via ARMA identification methods for prosthesis control purposes. IEEE Trans Syst Man Cybernet 1975; 2:252-258. 


\section{CONTROL OF MULTIFUNCTIONAL PROSTHETIC HANDS}

38. Graupe D, Salahi J, Kohn KH. Multifunctional prosthesis and orthosis control via microcomputer identification of temporal pattern differences in single-site myoelectric signals.J Biomed Eng 1982; 4:17-22.

39. Hudgins B, Parker P, Scott RN. A new strategy for multifunction myoelectric control. IEEE Trans Biomed Eng 1993; 40:82-94.

40. Englehart K, Hudgins B, Parker PA. A wavelet-based continuos classification scheme for multifunction myoelectric control. IEEE Trans Biomed Eng 2001; 48:302-311.

41. Zardoshti-Kermani M, Wheeler BC, Badie K, Hashemi RM. EMG feature evaluation for movement control of upper extremity prostheses. IEEE Trans Rehab Eng 1995; 3:324-333.

42. Park SH, Lee S-P. EMG pattern recognition based on artificial intelligence techniques. IEEE Trans Rehab Eng 1998; 6:400-405.

43. Han JS, Song WK, Kim JS, Bang WC, Lee H, Bien Z. New EMG pattern recognition based on soft computing techniques and its application to control a rehabilitation robotic arm. IIZUKA 2000 Int Conf Soft Comput, Iizuka, Fukuoka, Japan, October 1-4 2000; 1-4.

44. Micera S, Sabatini A, Dario P. On automatic identification of upper limb movements using small-sized training sets of EMG signal. Med Eng Phys 2000; 22:527-533.

45. Huang HP, Chiang CY. DSP-based controller for a multi-degree prosthetic hand. IEEE Intl Conf on Robotics and Automation (ICRA) 2000, San Francisco, California, April 24-28 2000; 1378-1383.

46. Kelly MF, Parker PA, Scott RN. The application of neural neyworks to myoelectric signal analysis: a preliminary study. IEEE Trans Biomed Eng 1990; 37:221-230.

47. Haykin S. Neural Networks: A Comprehensive Foudation. New York: MacMillian College Publishing, 1994.

48. Park SH, Lee SP. EMG pattern recognition based on artificial intelligence techniques. IEEE Trans Rehbil Eng 1998; 6:400-405.

49. Englehart K, Signal representation for classification of the transient myoelectric signal. $\mathrm{PhD}$ thesis, University of New Brunswick, Fredericton, Canada, 1998.

50. Karlsson S, Yu J, AKay M. Time-frequency analysis of myoelectric signals during dynamic contractions: a comparative study. IEEE Trans Biomed Eng 2000; 47:228-238.

51. Sparto PJ, Parnianpour M, Barria EA,Jagadeesh JM. Wavelet and short-time fourier transform analysis of electromyography for detection of back muscle fatigue. IEEE Trans Rehab Eng 2000; 8:433-436.

52. Pattichis CS, Pattichis MS. Time-scale analysis of motor unit action potentials. IEEE Trans Biomed Eng 1999; 46:1320-1329.

53. Akay M. Wavelet applications in medicine. IEEE Spectrum, 1997; 34:50-56.

54. Vetterli M, Kovacević J. Wavelets and Subband Coding. Englewood Cliffs, New Jersey: Prentice Hall, 1995.

55. Coifman RR,Wickerhauser MV.Entropy-based algorithms for best basis selection. IEEE Trans Inform Theory, 1992; 38:713-719.

56. Englehart K, Hudgins B, Parker PA, Stevenson M. Classification of the myoelectric signal using time-frequency based representations. Med Eng Phys 1999; 21:431-438.

57. Micera S, Sabatini A, Dario P, Rossi B. A hybrid approach for EMG pattern analyis for classification of arm movements using statistical and fuzzy techniques. Med Eng Phys 1999; 21: 303-311.

58. Kramer MA. Nonlinear principal component analysis using autoassociative neural networks. AIChE J 1991; 37:233-243.

59. Kohonen T. An introduction to neural computing. Neural Netw 1988; 1:3-16.

60. Micheli-Tzanakou E. Supervised and unsupervised pattern recognition: feature extraction and computational intelligence. Boca Raton, Florida: CRC Press, 2000.

61. Morita S, Kondo T, Ito K. Estimation of forearm movement from EMG signal and application to prosthetic hand control. IEEE Intl Conf on Robotics and Automation (ICRA), Seoul, May 21-26 2001; 3692-3697. 


\section{ZECCA ET AL.}

62. Christodoulou CI, Pattichis CS. Unsupervised pattern recognition for the classification of EMG signals. IEEE Trans Biomed Eng 1999; 46:169-178.

63. Santa-Cruz MC, Riso RR, Sepulveda F. Evaluation of neural network parameters towards enhanced recognition of naturally evoked EMG for prosthetic hand grasp control.IFESS 2000, Aalborg, Denmark, June 18-24 2000; 436-439.

64. Kosko B. Fuzzy Engineering. New York: Prentice Hall, 1996.

65. Mitra S, Hayashi Y. Neuro-fuzzy rule generation: Survey in soft computing framework. IEEE Trans Neural Netw 2000; 11:748-768.

66. Chan FHY, Yang YS, Lam FK, Zhang YT, Parker PA. Fuzzy EMG classification for prosthesis control. IEEE Trans Rehab Eng 2000; 8:305-311.

67. Tascillo A, Bourbakis N. Neural and fuzzy robotic hand control. IEEE Trans Syst Man Cybernet B 1999; 29.

68. Abe S, Lan M.A method for fuzzy rule extraction directly from numerical data and its application to pattern classification. IEEE Trans Fuzzy Syst 1995; 3:18-28.

69. Nishikawa D, Yu W, Yokoi H, Kakazu Y. EMG prosthetic hand controller discriminating ten motions using real-time learning method. IEEE IROS '99, Kyongju, South Korea, October 17-21 1999; 1592-1597.

70. Nishikawa D, Yu W, Yokoi H, Kakazu Y. Analyzing and discriminatin EMG signals using wavelet transform and real-time learning method. Artificial Neural Networks in Engineering Conference (ANNIE'99), St. Louis, Missouri, November 7-10 1999; 281-286.

71. Nishikawa D, Ishikawa Y, Yu W, Maruishi M, Watanabe I, Yokoi H, Mano Y, Kakazu Y. Online learning based EMG prosthetic hand. In: Electrophysiology and Kinesiology. Bologna, Italy: Mondiuzzi Editore, 2000; 575-580.

72. Fukuda O,Tsuji T, Ohtsuka A, Kaneko M.EMG-based human-robot interface for rehabilitation aid. Proc 1998 IEEE Intl Conf Rob Autom, Leuven, Belgium, May 16-20 1998; 3492-3497.

73. Netter FH. Anatomy, physiology and metabolic disorders. Atlas of Human Anatomy. vol. 8. Musculoskeletal System, Pt. 1. Ciba-Geigy Pharmaceutical Products, 1988.

74. Johansson R, Vallbo A. Tactile sensibility in the human hand: relative and absolute densities of four types of mechanoreceptive units in glabrous skin. J Physiol 1979; 286:405-422.

75. Vallbo AB,Johannson RS. Properties of cutaneous mechanoreceptors in the human hand related to touch sensation. Human Neurobiol 1984; 3:2-14.

76. Routhier F, Rancourt D, Gosselin CM. Design of a hand prosthesis based on kinematic principles. Proceedings of the Myoelectric Controls Powered Prosthesis Symposium, Fredericton, NB, Canada, 1995; 53-56.

77. Schulz S, Pylatiuk C, Bretthauer G. A new ultralight antropomorphic hand. IEEE Intl Conf on Robotics and Automation (ICRA) 2001, Seoul, May 21-26 2001; 2437-2441.

78. Speeter TH. Primitive based control of the Utah/MIT dextrous hand. Intl Conf on Robotics and Automation (ICRA), Sacramento, California, April 9-11 1991; 866-877.

79. Mason M, Salisbury J. Robot Hands and the Mechanics of Manipulation. Cambridge, Massachusetts: MIT Press, 1985.

80. Butterfaß J, Grebenstein M, Liu H, Hirzinger G. DLR-hand II: next generation of a dextrous robot hand. Intl Conf on Robotics and Automation (ICRA), Seoul, May 21-26 2001; 109-114.

81. Lovchik CS, Diftler MA. The robonaut hand: a dexterous robot hand for space. In: IEEE Intl Conf on Robotics and Automation (ICRA), Detroit, Michigan, May 10-15 1999; 907-912.

82. Fujii S, Nishikawa D, Yoko H. Development of a Prosthetic Hand Using Adaptable Control Method for Human Characteristics, vol. 5. Amsterdam: IOS Press, 1998; 360-376.

83. Williams WT. One-muscle infant's myoelectric control. J Assoc Children's Prosthet Orthot Clin 1989; 24.

84. Kelly MF, Parker PA, Scott RN. Myoelectric signal analysis using neural networks. IEEE Eng Med Biol 1990; 9:61-64.

85. Dorcas D, Scott RN. A three state myoelectric control. Med Biol Eng 1966; 4:367-372. 


\section{CONTROL OF MULTIFUNCTIONAL PROSTHETIC HANDS}

86. Kyberd PJ, Evans M, Winkel S. An intelligent anthropomorphic hand, with automatic grasp. Robotica 1998; 16:531-536.

87. Kyberd PJ, Evans M. Intelligent control of a prosthetic hand. 4th Eur Conf for the Advancement of Assistive Technology (AAATE'97), Thessaloniki, Greece, September 29-October 2 1997; $1-4$.

88. Eriksson L, Sebelius F, Balkenius C. Neural control of a virtual prosthesis. ICANN 98, Perspectives in Neural Computing. Niklasson L, Bodén L, Ziemke T, editors. Skövde, Sweden, September 2-4 1998; 905-910.

89. Kuiken T. Neuromuscular reorganization to improve the control of myoelectric prostheses. $9^{\text {th }}$ World Congr of the Intl Soc for Prosthetics and Orthotics (ISPO). Amsterdam, June 28-July 5 1998; 658.

90. Bonato P. Recent advancements in the analysis of dynamic EMG data. IEEE Eng Med Biol 2001; 20:29-32.

91. Montelius L, Sebelius F, Erikssson L, Holmberg H, Shouenbourg J, Danielsen N, Wallman L, Laurell T, Balkenius C. Pattern recognition of nerve signals using an artificial neural network. Proc of 18th Annual Intl Conf of the IEEE Engineering in Medicine and Biology Society, Amsterdam, October 31-November 3 1996; 1502-1503.

92. Dario P, Garzella P, Toro M, Micera S, Alavi M, Meyer U, Valderrama E, Sebastiani L, Ghelarducci B, Mazzoni C, Pastacaldi P. Neural interfaces for regenerated nerve stimulation and recording. IEEE Trans Rehabil Eng 1998; 6:353-363.

93. Riso RR. Strategies for providing upper extremity amputees with tactile and hand position feedback-moving closer the bionic arm. Technol Health Care 1999; 7:401-409.

94. Veltink PH. Sensory feedback in artificial control of human mobility. Technol Health Care 1999; 7:383-391.

95. Weingarden H, Zeilig G, Heruti R, Shemesh Y, Ohry A, Dar A, Katz D, Nathan R, Smith A. Hybrid functional electrical stimulation orthosis system for the upper limb: effects on spasticity in chronic stable hemiplegia. Am J Phys Med Rehabil 1998; 77:276-281.

96. Smith B, Tang Z, Pourmehdi M, Gazdik M, Buckett J, Peckham P. An externally powered, multichannel, implantable, stimulator-telemeter for control of paralyzed muscle. IEEE Trans Biom Eng 1998; 45:463-175. 
